\title{
The Endoscopic Removal of a Nasally Ectopic Premolar Tooth
}

\author{
Andrea Beech1, Simon Haworth'1, David Michael Thomas², Jeremy Farrier1 \\ ${ }^{1}$ Department of Oral and Maxillofacial Surgery, Gloucestershire Royal Hospital, Gloucestershire Hospitals NHS \\ Foundation Trust, Gloucester, UK \\ ${ }^{2}$ Department of Otolaryngology, Gloucestershire Royal Hospital, Gloucestershire Hospitals NHS Foundation \\ Trust, Gloucester, UK \\ Email: Andrea.Beech@glos.nhs.uk
}

Received 18 February 2015; accepted 25 May 2015; published 28 May 2015

Copyright (C) 2015 by authors and Scientific Research Publishing Inc.

This work is licensed under the Creative Commons Attribution International License (CC BY).

http://creativecommons.org/licenses/by/4.0/

(c) (i) Open Access

\begin{abstract}
Nasally ectopic teeth are rare, but can be a source of significant morbidity. Symptoms can include facial pain, nasolacrimal duct obstruction, fungal rhinitis, persistent rhinorrhea and episodic epistaxis. A nasally ectopic tooth should be considered as a differential diagnosis for a radiopaque lesion within the nasal cavity. This case illustrates the diagnosis and subsequent minimally traumatic recovery of a nasally ectopic tooth, believed to be an upper first premolar tooth, via an endoscopic approach.
\end{abstract}

\section{Keywords}

\section{Ectopic Tooth, Endscopic Removal, Nasal Tooth}

\section{Introduction}

Teeth or tooth like structures can develop in a wide range of locations. Supernumerary or supplemental teeth frequently develop in an ectopic location, however any permanent tooth can also form in an abnormal location. The most commonly seen ectopic teeth are the permanent canine teeth, the second premolar teeth and the third molar teeth. Ectopic teeth or tooth-like structures have been reported in sites as distant as the coronoid process, mandibular condyle [1], orbital floor, the maxillary sinus [2] or the nose.

Nasally ectopic teeth are rare, but have the potential to cause significant morbidity. Reports indicate that they can cause nasolacrimal duct obstruction [3], fungal rhinitis [4], facial pain, foul smelling rhinorrhea [5] and epistaxis [6] [7]. Clinically they could present as any of these complaints, or as a combination of several if not all. They may be palpable clinically or not depending on their exact position. In this article, we present a case report 
of a nasally ectopic tooth that had caused 30 years of foul smelling rhinorrhea. We aim to address the likely etiology of nasally ectopic teeth and their diagnosis. We also report the removal of a nasally ectopic tooth via an endoscopic approach which was simple and effective in this case.

\section{Case Report}

A 56-year-old lady was referred to the Oral and Maxillofacial Surgery department of a district general hospital complaining of left sided nasal discharge. She gave a history of 30 years of intermittent problems with her left nose, and 12 months of frequent green coloured nasal discharge. The patient noted that she had an increase in symptoms when she was suffering from any viral infection. She had been given several courses of broad-spectrum anti-biotics by her general medical practitioner which settled the nasal discharge, but then returned weeks later.

There was no history of maxillofacial trauma or surgery. The patient's medical history was unremarkable with the patient being generally fit and well. She had no allergies and took no regular medication. There was no history of any developmental disorder, nor any suspicion of an occult cleft. There was no known family history of a similar complaint or presentation. The patient did not drink alcohol and did not smoke.

Clinical examination was un-remarkable. There was no visible mass or foreign body, nothing abnormal to palpate and no nasal obstruction. A CT scan revealed a radiopaque mass in the left inferior meatus, adjacent to the septum. The lesion appeared uniformly radiopaque, with a radiodensity similar to that of cortical bone or dentine. The lesion appeared in close proximity or possibly in continuity with to the hard palate (Figure 1, Figure 2).

The initial differential diagnosis was that of an osteoma or calcified foreign body. After discussion with the department of Otolaryngology, arrangements were made for endoscopic retrieval under general anaesthesia.

The procedure was carried out as a joint case between Oral and Maxillofacial surgery and Otolaryngology. The mass was first visualized via nasendoscopy and mobilized via cautiousmanipulation, before being delivered intact via the left naris. No bone removal was required, and satisfactory haemostasis occurred after gentle application of a haemostatic sponge for a short period of time.

The retrieved object had the macroscopic appearance of a premolar tooth. The crown morphology and cuspal architecture are grossly normal for a premolar tooth, but the roots are poorly developed (Figure 3).

Histological investigation confirmed the intra-operative clinical impression of a nasally ectopic tooth.

Clinical examination of the oral cavity revealed an absent upper left first premolar tooth (UL4), with nohistory of previous exodontia. This would tend to support the view that the object retrieved was anectopic permanent upper first premolar tooth rather than a supplemental, supernumerary or primarytooth.

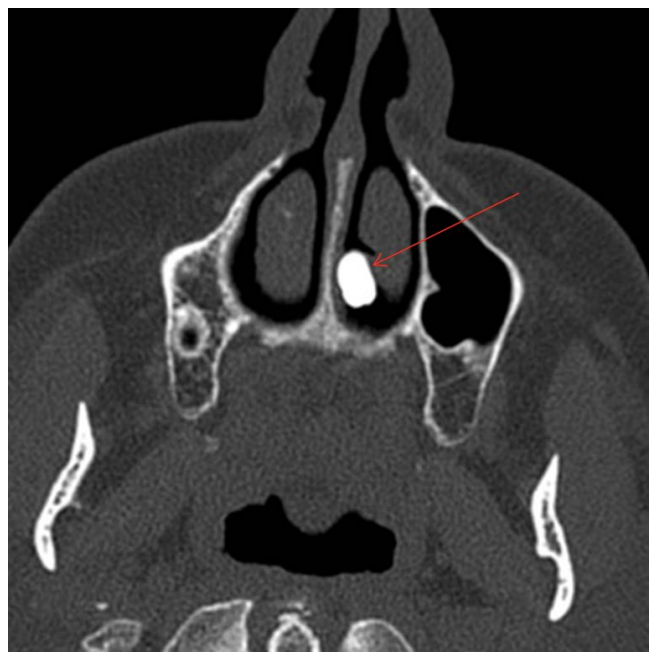

Figure 1. Radiopaque lesion visualized on coronal CT imaging. Red arrow indicating the radiopaque lesion. Source: Pre-op CT scan. Used with patient permission. 


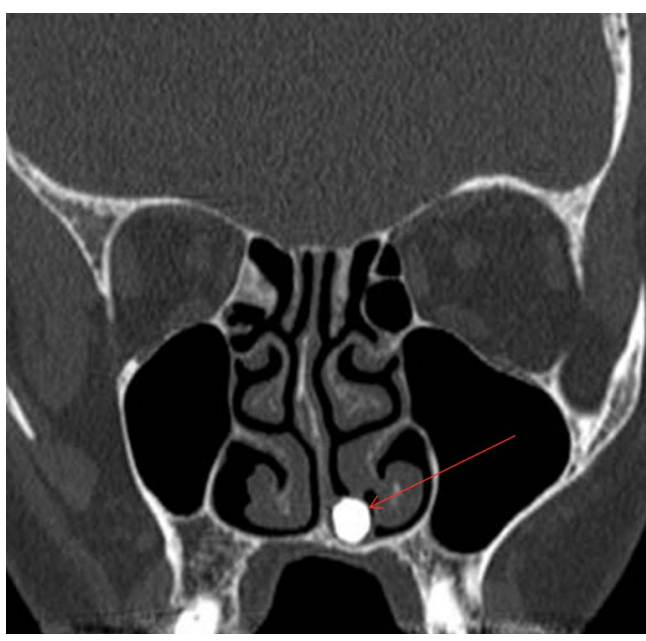

Figure 2. Radiopaque lesion visualized on axial CT imaging. Red arrow indicating the radiopaque lesion. Source: Pre-op CT scan. Used with patient permission.

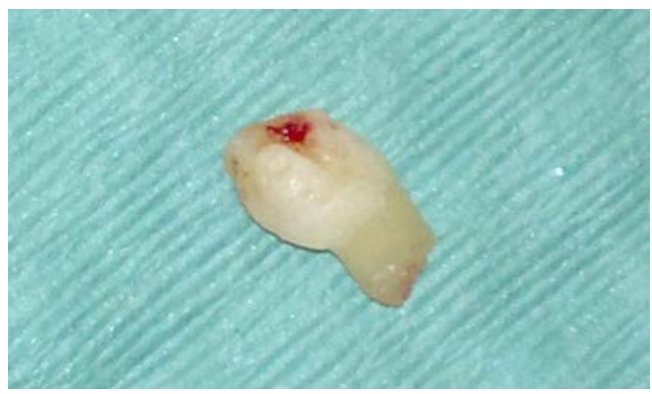

Figure 3. Photograph of tooth immediately after removal. Source: Author. Used with patient permission.

At a review appointment 6 weeks after the procedure, the patient reported complete resolution of hersymptoms and had not experienced any post-operative epistaxis or other complications.

\section{Discussion}

Although rare, ectopic teeth should be considered as a differential diagnosis for a radiopaque lesion in the nasal cavity. At the time of initial presentation, and subsequent CT scanning, the differential diagnosis in this case was either an osteoma or calcified foreign body with a nasal tooth not being initially suspected. Other differential diagnoses could include a rhinolith, a granulomatous infection [8], a calcified polyp, aendochondromaor malignant tumours such as chondrosarcoma or osteosarcoma [9].

In our search of the available literature the majority of previously described nasal teeth have been implicated as supernumery or supplemental [9]. This nasally ectopic tooth was not considered to be a supernumery tooth as the upper left permanent first premolar tooth was missing intra-orally. This is a less common tooth to be found in an ectopic position compared to other permanent teeth i.e. the canine, second premolar and third molar teeth.

It is generally accepted that the ectopic growth and positioning of a tooth is not well understood. One theory is that an ectopic tooth can develop when there is an abnormal tissue interaction to disrupt the process of the complex interaction occurring between the oral epithelium and underlying mesenchymal tissue in normal tooth development [10]. Other evidence has implicated the development of dentigerous cysts in teeth ectopically positioned in the maxillary sinus [2], but none to implicate this disease process in nasally ectopic teeth. We found no 
surrounding cystic mass intra-operatively in this case.

Other possible causes of an ectopic tooth could potentially be traumatic, iatrogenic [11], a developmental disturbance/anomaly or genetic predisposition [1]. The patient had received no previous medical treatment and had no history of trauma. She also had no known developmental disorder and was unaware of any relevant family history. We consider the nasally ectopic tooth in this case to be best regarded as having an idiopathic cause.

Literature supports removal of these teeth, as they can cause significant morbidity. This case and the existing literature illustrates that endoscopic retrieval of these teeth can be safe and effective, with no significant complications [12]. Endoscopic removal has the specific advantages of being minimally invasive, offering clear visualization by good illumination and allowing a short an aesthetic time.

In this case early suspicion of a nasally ectoptic tooth was not considered most likely because the patient was not referred to the department of Oral and Maxillofacial Surgery department in the first instance. An examination of the oral cavity during treatment planning would alert the clinician to the missing upper left first premolar tooth, and hence make them highly suspicious of potentially ectopic position. The missing tooth was noted after the endoscopic procedure. Treatment however would not have changed, but it is an important learning point for the reader to consider in a similar case presentation.

\section{Conclusion}

We conclude that the removal of a nasally ectopic tooth with an endoscopic approach is very effective and we recommend it in the management of this rare entity. It is important that case history taking should include previous maxillofacial trauma and exodontia for a suspected nasally ectopic tooth. Something we did not originally consider in this case. Examination of the oral cavity can provide clues as to the origin and nature of a mass present in the nose when reaching a provisional diagnosis.

\section{Patient Permission}

The patient has kindly granted their permission for publication and use of radiographic images.

\section{References}

[1] Gadre, K.S. and Waknis, P. (2010) Intra-Oral Removal of Ectopic Third Molar in Mandibular Condyle. International Journal of Oral and Maxillofacial Surgery, 39, 294-296. http://dx.doi.org/10.1016/j.ijom.2009.10.002

[2] Ramanojam, S., Halli, R., Hebbale, M. and Bhardwaj, S. (2013) Ectopic Tooth in Maxillary Sinus: Case Series. Annals of Maxillofacial Surgery, 3, 89-92.

[3] Alexandrakis, G., Hubbell, R.N. and Aitken, P.A. (2000) Nasolacrimal Duct Obstruction Secondary to Ectopicteeth. Ophthalmology, 107, 189-192. http://dx.doi.org/10.1016/S0161-6420(99)00023-8

[4] Chen, A., Huang, J.K., Cheng, S.J. and Sheu, C.Y. (2002) Nasal Teeth: Report of Three Cases. American Journal of Neuroradiology, 23, 671-673.

[5] Lee, J.H. (2006) A Nasal Tooth Associated with Septal Perforation: A Rare Occurrence. European Archives of Otorhinolaryngology, 263, 1055-1056. http://dx.doi.org/10.1007/s00405-006-0107-X

[6] Krishnan, B., Parida, P., Gopalakrishnan, S. and Satyparakash, M.V.S. (2013) An Unusual Cause of Epistaxis in a Young Patient: The Supernumerary Nasal Tooth. Oral and Maxillofacial Surgery, 17, 315-317. http://dx.doi.org/10.1007/s10006-013-0393-6

[7] Al Dhafeeri, H., Kavarodi, A., Al Shaikh, K., Bukhari, A., Al Hussain, O. and El Baramawy, A. (2014) Recurrent Epistaxis Caused by an Intranasal Supernumery Tooth in a Young Adult. American Journal of Case Reports, 15, 291-293. http://dx.doi.org/10.12659/AJCR.890710

[8] Martison, F.D. and Cockshott, W.P. (1972) Ectopic Nasal Dentition. Clinical Radiology, 23, 451-454. http://dx.doi.org/10.1016/S0009-9260(72)80015-1

[9] Arunkumar, J.S., Prasad, K.C. and Shanthi, N. (2007) Nasal Teeth: A Case Report. Indian Journal of Otolaryngology and Head Neck Surgery, 59, 197-198.

[10] Srinivasa Prasad, T., Sujatha, G., Niazi, T.M. and Rajesh, P. (2007) Dentigerous Cyst Associated with an Ectopic Third Molar in the Maxillary Sinus: A Rare Entity. Indian Journal of Dental Research, 18, 141-143. http://dx.doi.org/10.4103/0970-9290.33793

[11] Lamb, J.F., Husein, O.F. and Spiess, A.C. (2009) Ectopic Molar in the Maxillary Sinus Precipitating a Mucocele: A 
Case Report and Literature Review. Ear, Nose Throat Journal, 88, E6-E11.

[12] Sanei-Moghaddam, A., Hyde, N. and Williamson, P. (2009) Endoscopic Removal of a Supernumerary Tooth from the Nasal Cavity in an Adult. British Journal of Oral and Maxillofacial Surgery, 47, 484-485.

http://dx.doi.org/10.1016/j.bjoms.2009.04.036 